\title{
OUTDOOR STAND-OFF INTERROGATION OF FISSIONABLE MATERIAL WITH A HYBRID CODED IMAGING SYSTEM *
}

\author{
A.L. Hutcheson ${ }^{\xi}$, B.F. Phlips, E.A. Wulf \\ Radiation Detection Section (Code 7654), U.S. Naval Research Laboratory, 4555 Overlook Ave. SW \\ Washington, DC, USA \\ B.V. Weber \\ Pulsed Power Division (Code 6770), U.S. Naval Research Laboratory, 4555 Overlook Ave. SW \\ Washington, DC, USA \\ R.S. Woolf \\ National Research Council Postdoctoral Fellow, 500 Fifth St. NW \\ Washington, DC, USA
}

\section{Abstract}

A hybrid coded imaging and detection system developed at the U.S. Naval Research Laboratory (NRL) was used for active interrogation measurements with pulsed bremsstrahlung at the Hermes-III facility at Sandia National Laboratories, Albuquerque. This work follows previous experiments performed in 2011 [1] and explores different targets and system conditions to the previous work. The techniques used and challenges encountered during this work are described.

\section{INTRODUCTION}

Passive detection of shielded special nuclear material (SNM) is a difficult problem, particularly at stand-off distances. Even moderate amounts of shielding can be sufficient to successfully mask the naturally occurring gamma rays and/or neutrons emitted by SNM. Alternatively, active interrogation methods can be used to probe materials through shielding and generate a unique and measurable signal in fissionable material; in these methods, a source of interrogating radiation is used to induce fission in the fissionable material, resulting in the increased emission of gamma rays and/or neutrons. This increased emission can be separated into "prompt" (emitted less than $1 \mu \mathrm{s}$ after interrogation) and "delayed" (emitted more than $1 \mu \mathrm{s}$ after interrogation) signals. However, the use of interrogating radiation can also produce higher background radiation levels due to activation of the shielding or surrounding materials. Both the induced signal from the fissionable target and the active background radiation level change with the type and energy of the interrogating radiation used. The challenge is therefore to determine the optimal species and energy of interrogating radiation as well as the optimal time window after activation in which to measure the induced signal. Previous investigations into the use of bremsstrahlung interrogating radiation have focused on intense, single bremsstrahlung pulses on indoor targets with standoff distances on the order of a few meters [2] or on investigating prompt emission signatures between repeated pulses of lower-intensity bremsstrahlung radiation [3]. In contrast, the work described herein studies the delayed signals produced by a single intense interrogating pulse in an outdoor setting with standoff distances on the order of tens of meters.

\section{EXPERIMENT}

In September and October of 2012, measurements were performed at the Hermes-III facility at Sandia National Laboratories, Albuquerque, to study the effects of low- and high- $Z$ shielding on the active interrogation of fissionable materials. The Hermes-III facility is a $16 \mathrm{MV}$, $500 \mathrm{kA}$ pulsed electron beam accelerator capable of producing $30 \mathrm{~ns}$ pulses of bremsstrahlung radiation into an outdoor test cell [4]. The fissionable targets in question included a $30 \times 30 \times 2.5 \mathrm{~cm}$ depleted uranium (DU) plate, a $1000 \mathrm{~cm}^{3}$ DU cube, and a $10 \times 10 \times 2 \mathrm{~cm}$ low-enriched uranium (LEU) plate. The target was located outdoors approximately $18 \mathrm{~m}$ away from the $\mathrm{x}$-ray source. Target shielding materials included lead ( $5 \mathrm{~cm}$ thick), steel $(7.5$ $\mathrm{cm}$ thick), and 2\%-borated polyethylene (BPE) (10 and 25 cm thick). The Hermes-III accelerator was run in half-, "medium"-, and full-machine modes to produce

\footnotetext{
* Work conducted under the auspices of the Defense Threat Reduction Agency.

₹ email: anthony.hutcheson@nrl.navy.mil
} 
bremsstrahlung pulses with approximate endpoint energies of 8,12 , and $16 \mathrm{MeV}$, respectively.

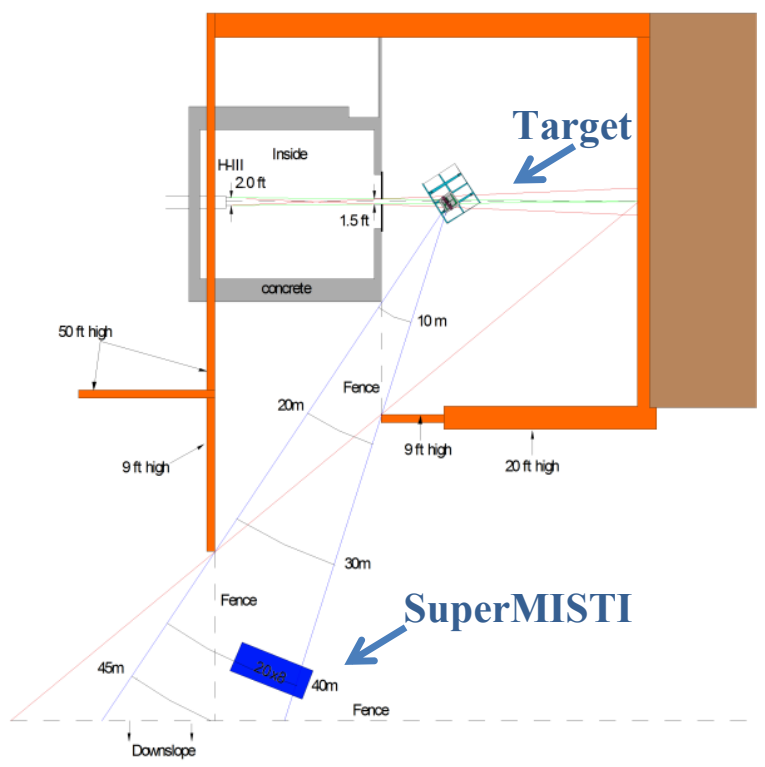

Figure 1. Layout of the Hermes-III test area.

The induced signals from the targets were detected by the imaging/localization subsystem of the SuperMISTI hybrid detection system [5], which was located approximately $40 \mathrm{~m}$ from the target location (see Figure 1). The SuperMISTI imaging/localization subsystem consists of $78 \mathrm{NaI}$ detectors $(\varnothing 15 \times 15 \mathrm{~cm})$ mounted into a $6 \times 13$ array on one side of a $20 \mathrm{ft}$ refrigerated ISO container. The entire array is shielded from above, below, behind, and the sides by $2.5 \mathrm{~cm}$ of lead; in addition, lead flooring $(2.5 \mathrm{~cm}$ thick) provides further shielding from background radiation originating from below. Mounted on the opposite side of the container is a $12 \times 27$ pseudorandom mask that comprises 162 lead blocks $(15 \times 15 \times 5 \mathrm{~cm})$. The mask array is $50 \%$ open, $50 \%$ masked. The pattern of counts in the NaI detectors as a result of the lead mask can be deconvolved to produce a coded image of a gamma-ray source. An optical camera mounted on the exterior of the ISO container provides an image onto which this coded image is then overlaid; such a combined image is shown in Figure 2.

For the active interrogation measurements at HermesIII, only gamma rays with energies between 3 and $7 \mathrm{MeV}$ were considered. This energy range was chosen for three reasons. First, there is very little natural background gamma radiation above approximately $2.6 \mathrm{MeV}$. Second, the two dominant sources of activation counts in the NaI detectors result from thermal neutron activation of the aluminum casing of the detectors (resulting in a 1.779 $\mathrm{MeV}$ gamma ray) and of the iodine in the detector itself (a continuum ranging from $443 \mathrm{keV}$ to $2.562 \mathrm{MeV}$ due to beta and gamma decay). Third, a unique signature that spans energies greater than $3 \mathrm{MeV}$ is emitted by the beta decay of the short-lived fission products of fissionable materials [6]; this signature is absent when nonfissionable materials are interrogated. These three features are clearly evident in the spectra shown in Figure 3.

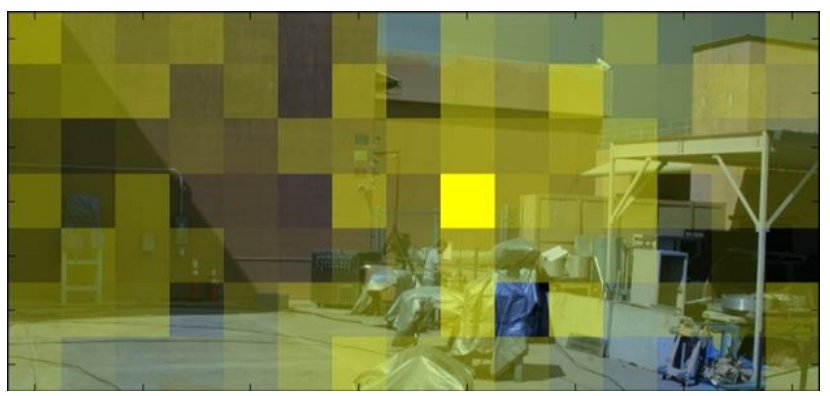

Figure 2. Coded image produced from 3-7 MeV gamma emitted from interrogated DU target overlaid on optical camera image. The "hot" pixel denotes the DU target location.

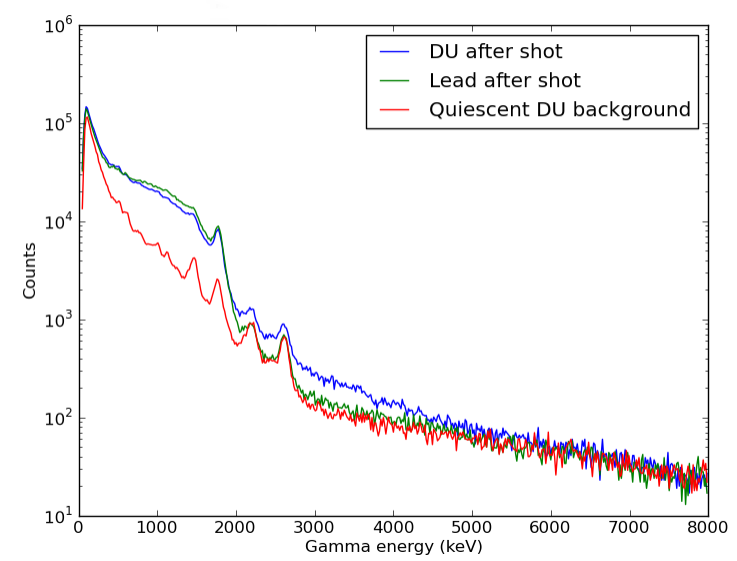

Figure 3. Comparison of gamma spectra measured in $\mathrm{NaI}$ detectors for DU and lead targets. Quiescent background denotes spectra for target without interrogating radiation.

In addition to the gamma imaging detectors, a complement of $\mathrm{six}^{3} \mathrm{He}$ detectors $(\varnothing 15 \times 64 \mathrm{~cm} ; 2.66 \mathrm{~atm})$ were integrated into the ISO container for neutron detection. The ${ }^{3} \mathrm{He}$ detectors were each surrounded by an inner layer $(2.5 \mathrm{~cm})$ of high-density polyethylene followed by an additional outer layer $(2.5 \mathrm{~cm})$ of BPE. The net effect of this two-layer moderation was to lower the detector efficiency for thermal neutron signals while boosting the efficiency for fast neutrons.

\section{RESULTS}

\section{A. Active gamma background}

A rate plot of the 3-7 MeV gamma rays measured by the $\mathrm{NaI}$ detectors during 8, 12, and $16 \mathrm{MeV}$ bremsstrahlung pulses with no target is shown in Figure 4. The gamma ray counts in this plot represent the active gamma background associated with these pulses. Note that both the amplitude and the duration of these counts 
increase as the endpoint energy of the bremsstrahlung pulse increases. The duration of these active background counts indicate that any prompt gamma-ray measurements must contend with a significant active background; however, for signals more than $0.5 \mathrm{~s}$ after the bremsstrahlung pulse, the active background has essentially decayed back to natural background levels.

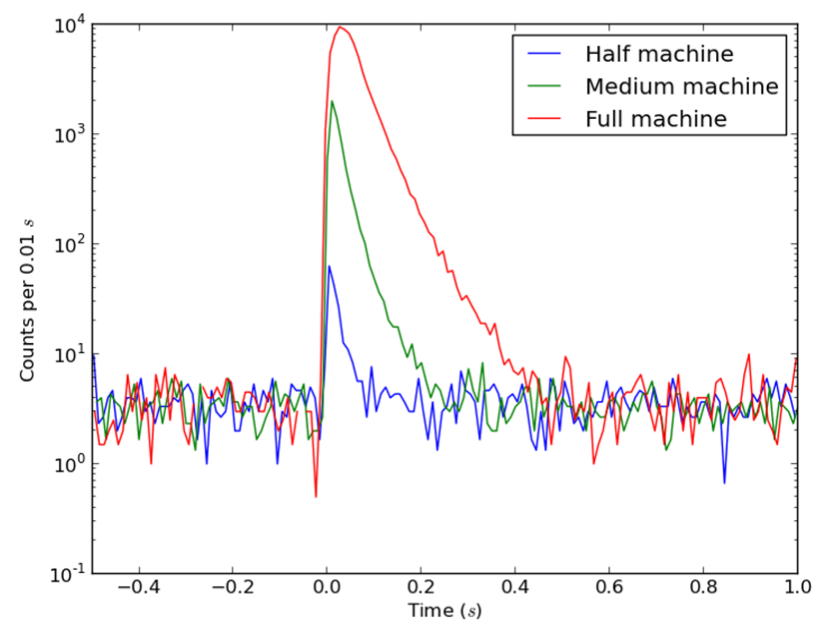

Figure 4. Rate plot of 3-7 MeV gamma rays produced during 8 (half machine), 12 (medium machine), and 16 (full machine) $\mathrm{MeV}$ bremsstrahlung pulses with no target.

The associated spectrum for the active background gamma rays is a continuum of counts spanning the entire energy range. The energy spectra for different $100 \mathrm{~ms}$ time bins after a full-machine pulse is shown in Figure 5. Note the lack of any discrete peaks and the enhanced continuum of counts that decays over time.

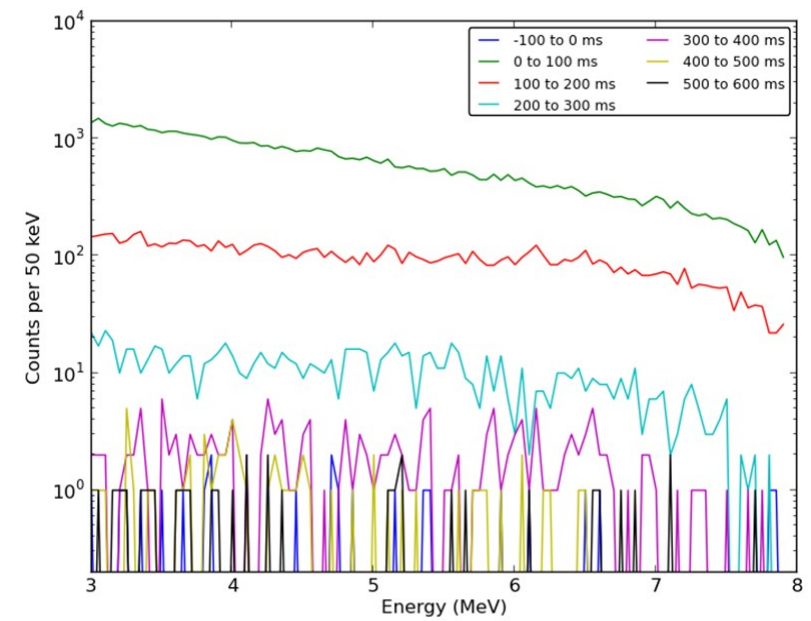

Figure 5. Spectra of high-energy active background gamma rays produced after a $16 \mathrm{MeV}$ bremsstrahlung pulse.

When a coded image of these active background counts is produce, the resulting image indicates that the source of these high-energy gamma rays is localized in the outdoor test cell. This result indicates that these high-energy gamma rays result from the activation of environmental materials by the interrogating pulse. Further measurements are needed to determine if any particular materials in the environment are responsible for this background signature.

\section{B. Active neutron background}

A rate plot of the neutrons measured by the ${ }^{3} \mathrm{He}$ detectors during 8,12 , and $16 \mathrm{MeV}$ bremsstrahlung pulses with no target is shown in Figure 6. The neutron counts in this plot represent the active neutron background associated with these pulses. Note that, similar to rate plots for the gamma rays, both the amplitude and the duration of the neutron counts increase as the endpoint energy of the bremsstrahlung pulse increases. However, for each bremsstrahlung energy, the duration of the associated neutron active background is shorter than that of the associated gamma active background. Although there is currently no imaging capability for neutrons in the SuperMISTI system, it is assumed that the source of these neutrons, like that of the active gamma background, is located in the outdoor test cell and is associated with photonuclear reactions in environmental materials located therein.

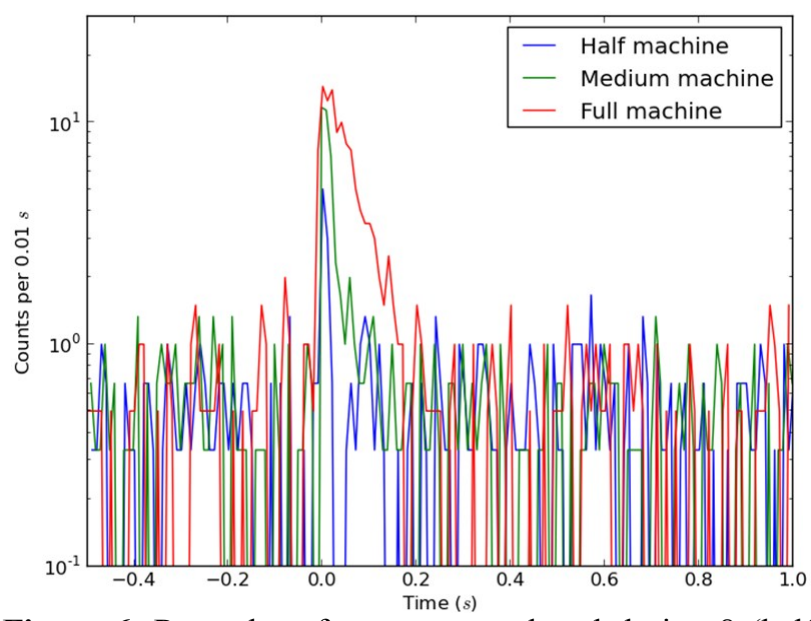

Figure 6. Rate plot of neutrons produced during 8 (half machine), 12 (medium machine), and 16 (full machine) $\mathrm{MeV}$ bremsstrahlung pulses with no target.

\section{Detection of fissionable materials}

Analysis is currently underway to determine the effects of the different shielding configurations used and the endpoint energy of interrogating bremsstrahlung radiation on the detection of fission gamma rays and neutrons. However, early results indicate that the best detections were seen for gammas produced greater than 0.5 seconds after full-machine bremsstrahlung shots. Preliminary gamma detection and imaging results are shown in Figures 7 and 8 for full-machine shots on, respectively, DU and lead targets. Significant full-machine neutron detections were also seen after 0.5 s. For mediummachine shots, gamma detections were possible at earlier 
times ( $0.3 \mathrm{~s}$ after the shots) but were weaker. Further analysis is required for half-machine gamma detections and medium- and half-machine neutron detections.
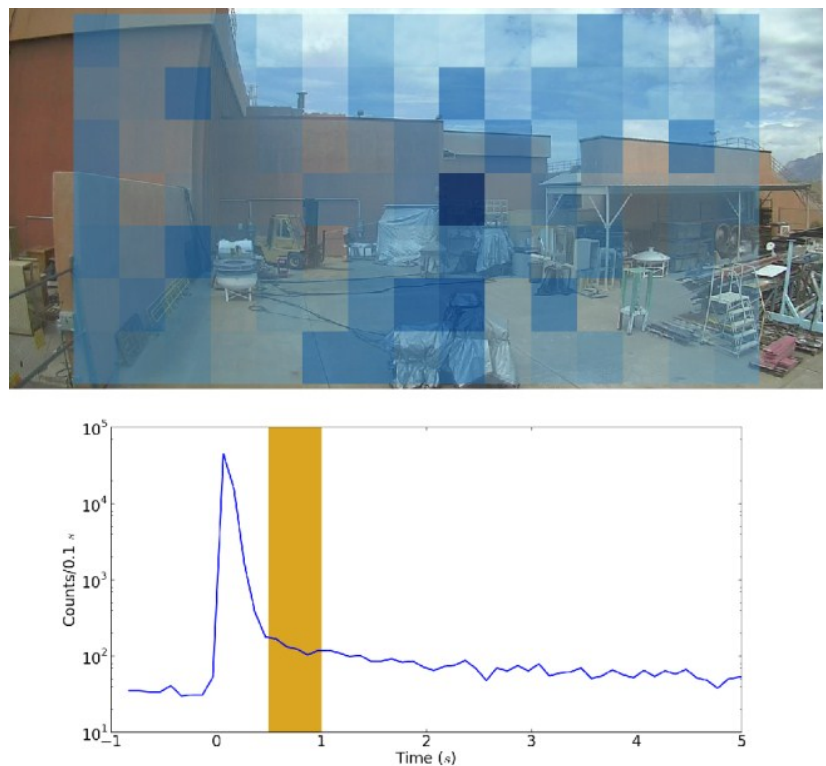

Figure 7. (top) Coded image of 3-7 MeV gammas detected $0.5-1.0 \mathrm{~s}$ after a $16 \mathrm{MeV}$ bremsstrahlung pulse on a DU target. (bottom) Rate plot of the 3-7 MeV gammas for this shot. The time window used to produce the above image is highlighted in orange.
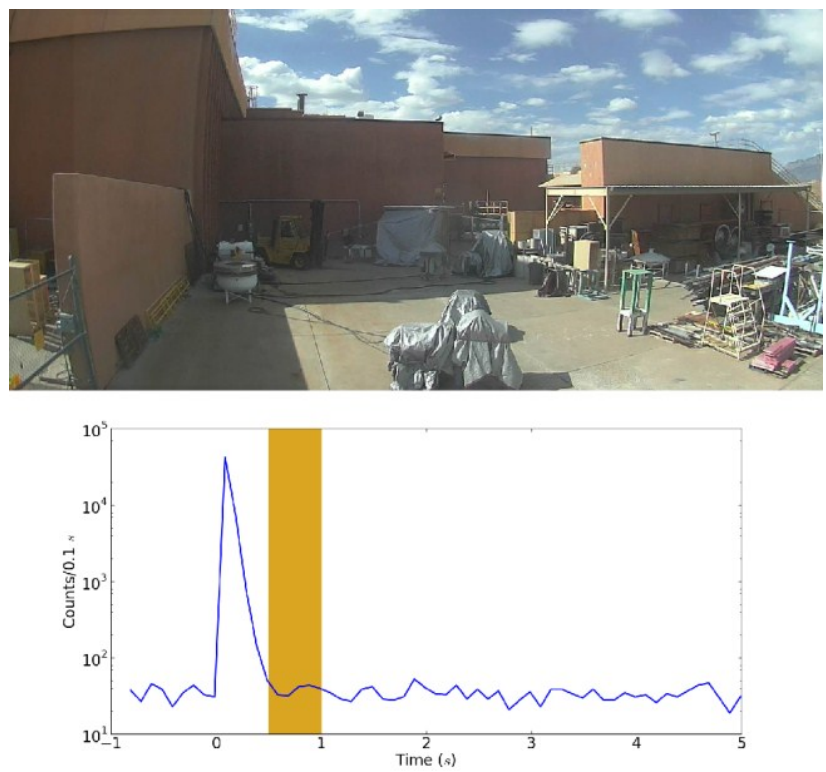

Figure 8. (top) Coded image of 3-7 $\mathrm{MeV}$ gammas detected $0.5-1.0 \mathrm{~s}$ after a $16 \mathrm{MeV}$ bremsstrahlung pulse on a lead target. (bottom) Rate plot of the 3-7 MeV gammas for this shot. The time window used to produce the above image is highlighted in orange.

\section{SUMMARY}

Active interrogation of fissionable materials has been investigated with the SuperMISTI imaging/localization subsystem at the Hermes-III facility at Sandia National Laboratories, Albuquerque. The detection of gamma rays and neutrons emitted from fissionable materials due to bremsstrahlung-induced photofission has been studied at a stand-off distance of $40 \mathrm{~m}$. Gamma and neutron active background signatures with durations of up to $0.5 \mathrm{~s}$ have been identified, and unique detection of fissionable materials has been demonstrated. Detection was best for delayed gamma rays induced by an interrogating pulse with a $16 \mathrm{MeV}$ endpoint energy. Analysis is currently underway to determine the effects of different shielding configurations on the detection of fissionable materials in this environment.

\section{ACKNOWLEDGEMENT}

The authors of this work gratefully acknowledge the funding support from the Defense Threat Reduction Agency for this project.

\section{REFERENCES}

[1] T.J. Renk et al., "Use of pulsed bremsstrahlung excitation on Hermes-III for investigation of active detection of fissionable material," in Plasma Science (ICOPS), 2012 Abstracts IEEE International Conference on, 2012, p. 6D-1.

[2] S.L. Jackson et al., "Detectors for intense, pulsed active detection," Nuclear Science Symposium Conference Record (NSS/MIC), 2010 IEEE, 2010, p. 516.

[3] E.T.E. Reedy, S.J. Thompson and A.W. Hunt, “The detection of delayed $\gamma$-rays between intense bremsstrahlung pulses for discriminating fissionable from non-fissionable materials," Nuclear Instruments and Methods in Physics Research Section A, vol. 606, pp. 811-815, Jul. 2009.

[4] J.J. Ramirez et al., "Performance of the Hermes-III gamma ray simulator," in Pulsed Power Conference, 1989. $7^{\text {th }}, 1898$, p. 26.

[5] E.A. Wulf, A.L. Hutcheson, B.F. Phlips, L.J. Mitchell and B.E. Leas, "Stand-off detection with an active interrogation photon beam," Nuclear Science Symposium and Medical Imaging Conference (NSS/MIC), 2011 IEEE, 2011, p. 315.

[6] E.B. Norman et al., "Signatures of fissile materials: high-energy $v$ rays following fission", Nuclear 
Instruments and Methods in Physics Research Section A, vol. 521, pp. 608-610, Apr. 2004. 